\title{
ASPECTOS GERAIS SOBRE A ALIMENTAÇÃO DO CANGULO, BALISTES VETULA LINNAEUS, 1758 (PISCES - BALISTIDAE) NO ESTADO DE PERNAMBUCO - BRASIL ${ }^{1}$
}

\author{
Técio Luiz Macêdo Costa ${ }^{2}$ \\ Antônio de Lemos Vasconcelos Filho ${ }^{3}$ \\ Elza Maria Braga Galiza-Viana ${ }^{3}$
}

\section{ABSTRACT}

The Balistidae, especially Balistes vetula are commom fishes in tropical waters from Northeastern Brazil. Studies about these fishes captured at the coastal area of Recife between Pina; Boa Viagem, Piedade and Candeias beaches were carried out in order to determine the diet, and to verify a possible diet diversification between the sexes, and times of the year. Males and females had a food diet consisting mostly of molluscs belonging the classes Scaphopoda, Bivalvia, Gastropoda and crustaceans and fishes. Echinoderms, foraminifera, bryozoa, sponges, polychdete worms, coelenterates and brachiopods were also observed in smaller proportions. Both males and females had a carnivorous benthic diet. Growth and changes in the seasons were not reflected by changes in the diet.

\section{INTRODUÇÃO}

Os peixes Balsistídeos, devido a sua ampla distribuição geográfica e a importância que algumas de suas espécies apresentam, têm, em vários países, servido como tema para um considerável número de trabalhos científicos.

Autores como Jordan \& Evermann (1896), Ribeiro (1915), Santos (1952), Herald (1962), Cenvigon (1966), Greenwood et alii (1966), Randall (1968), Soares Filho (1980), Menezes (1981), Moura (1981), Soares (1981), entre outros, têm realizado estudos importantes acerca da biologia, posição

\footnotetext{
1 Trabalho apresentado no XI Congresso Brasileiro de Loologia, realizado em Belém-PA, durante o período de 12 a 17 de fevereiro de 1984 .

2 Departamento de Pesca da UFRPE

3 Departamento de Oceanografia da UFPE, Bolsista do CNPq.
} 
sistemática, distribuição geográfica do Balistes vetula, inclusive dos hábitos alimentares.

A distribuição geográfica do cangulo, segundo Smith (1952) abrange o Atlântico Tropical e Oceano Índico; segundo Santos (1952) ocorre desde Massachusetts, América do Norte, até Angra dos Reis; e segundo Cervigon (1966), em ambos os lados do Atlântico, sendo que no Atlântico Ocidental ocorre desde Rhode Island (BUA) até o Brasil.

Sabe-se que o hábito alimentar é uma característica específica decorrente quer das condições ambientais, quer daquelas próprias da espécie. Por outro lado, além de ser fundamental em pesquisas de auto-ecologia, constitui também um meio de se obter informação a respeito da estrutura trófica do ecossistema, ao qual a espécie pertence.

No Nordeste do Brasil já foram realizados diversos estudos visando o conhecimento dos hábitos alimentares dos peixes: Barroso $(1965,1965 \mathrm{a})$, estudando a alimentação do pargo, Lutjanus aya Bloch, e o xaréu preto, Caranx lugubris Poey, verificou que ambos possuem um hábito alimentar zoófago; com relação ao peixe voador, Hirundicthys affinis Gunther, Barroso (1967) verificou que sua dieta alimentar é fito-zoófaga, devido à presença de algas, peixes, moluscos e insetos encontrados em seus estômagos: Menezes (1968), no Ceará, estudando a alimentação do camurupim, Tarpon atlanticus Valenciennes, concluiu que esse peixe possui um hábito alimentar zoófago; Menezes (1969), ao estudar a alimentação da cavala, Scomberomorus cavalla Cuvier, nas águas costeiras do Ceará, verificou que a mesma possui um hábito alimentar carnívoro; ainda no Ceará, Menezes (1970) verificou que a serra, Scomberomorus maculatus Mitchill, possui uma dieta alimentar também camívora; Furtado \& Menezes (1972), ao estudarem, no Nordeste brasileiro, a alimentação do pargo, Lutjanus purpureus Poey, concluíram que o mesmo é um peixe camívoro de hábitos bentônico e pelágico; Silva \& Vasconcelos Filho (1972), ao estudarem os camorins, Centropomus parallelus Poey e Centropomus undecimalis Bloch, concluíram serem peixes que apresentam uma dieta alimentar carnívora, constando essencialmente de crustáceos e peixes; Eskinazi-Leça et alii (1976), verificaram que os Mugilídeos demonstraram um hábito alimentar iliófago, devido a sua preferência alimentar ser diatomáceas e cianofíceas, principalmente aquelas do fitobentos; Menezes \& Aragão (1977), no Ceará, estudando a biologia do bonito, Euthynnus alletteratus Rafinesque, encontraram nos estômagos: peixes voadores, sardinhas, moréias e cangulos, no bolo alimentar; Vasconcelos Filho (1979) determinou a dieta alimentar da sardinhabandeira capturada ao longo do Canal de Santa Cruz - PE, como sendo um peixe planctófago; Vasconcelos Filho (1980), estudando a alimentação do carapicu, Eucinostomus spp., no Canal de Santa Cruz-PE, determinou uma dieta predominantemente zoófaga, para os mesmos; Vasconcelos Filho \& Galiza (1980), em Pemambuco, estudando os hábitos alimentares dos peixes centropomídeos cultivados em viveiros da região de Itamaracá, veri- 
ficaram um hábito alimentar eminentemente carnívoro; Guedes \& Vasconcelos Filho (1980) estudando, em Pernambuco, a alimentação dos bagres branco e amarelo, consideraram o hábito alimentar desses peixes como zoófago, em virtude da grande quantidade de animais encontrados em seus estômagos; Vasconcelos Filho et alii (1981), estudando a alimentação da carapeba listrada, Eugerres spp., e da carapeba prateada, Diapterus spp., provenientes do Canal de Santa Cruz, demonstraram possuir um hábito alimentar fito-zoófago, devido a grande quantidade de animais e vegetais superiores, microalgas, além de outros organismos menos comuns no bolo alimentar; Menezes (1981), estudan do a alimentação do cangulo, evidenciou que esse peixe possui uma alimentação camívora, essencialmente bentônica nos jovens e mista nos adultos.

Em Pernambuco, a participação do cangulo, Balistes vetula (Fig. 1), na pesca artesanal é de grande relevância, contribuindo assim para o enriquecimento da dieta da população de baixo poder aquisitivo, carente de proteína de origem animal.

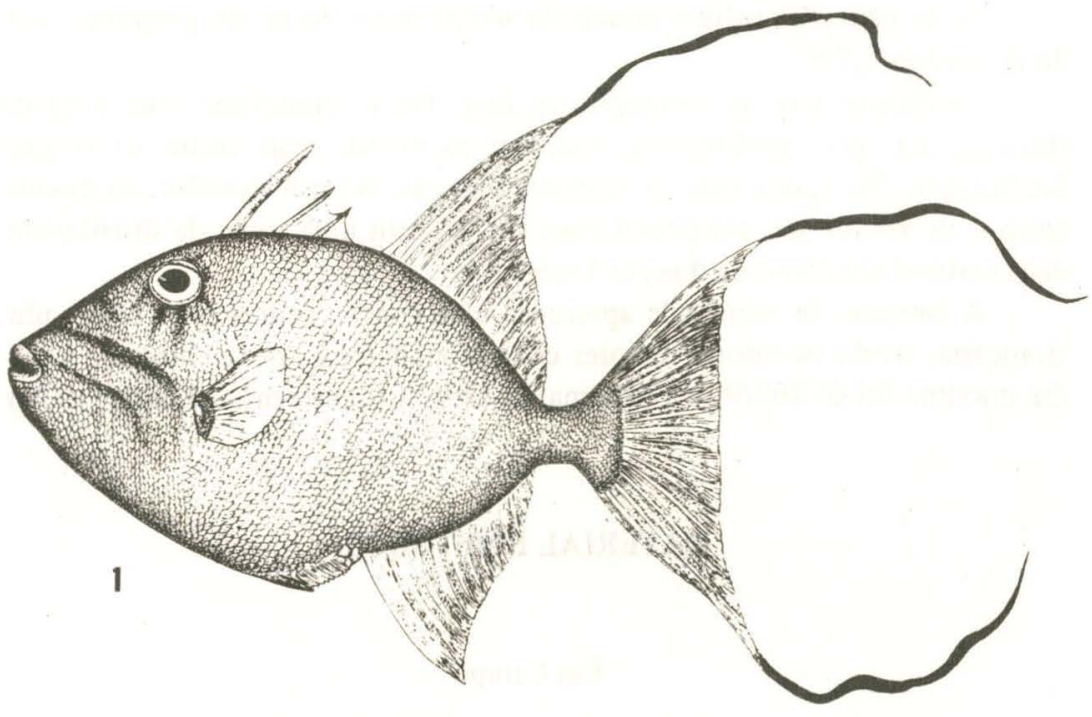

Fig. 1: Vista lateral do cangulo, Balistes vetula Linnaeus, 1758. 
Estudos realizados sobre a população e reprodução do cangulo, Balistes vetula, no Estado de Pernambuco, demonstraram serem esses peixes portadores de um dimorfismo sexual, ou seja, as fêmeas crescem mais que os machos (Soares, 1981).

Visto ao exposto, resolveu-se estudar o conteúdo gástrico da porção anterior do tubo digestivo desses indivíduos, tisando a obtenção de informações detalhadas sobre os seus hábitos alimentares, além de verificar uma possível diversificação alimentar quanto aos sexos e comprimento desses peixes.

\section{DESCRIÇÃO DA ẢREA}

A área em que foram coletados os exemplares situa-se no litoral sul do Estado de Pernambuco, a uma distância de até 20 milhas da costa, abrangendo a região do Pina até Barra das Jangadas, correspondente às latitudes 805'S e 8014'S (Fig. 2).

Esta área se localiza na faixa de clima tropical atlântico, com uma temperatura média próxima a $26^{\circ} \mathrm{C}$.

A distribuição pluviométrica define duas estações: uma seca pouco pronunciada e outra chuvosa, esta sob influência de frentes frias oriundas do sul.

Já as variações médias anuais de temperatura do ar são pequenas, sendo de apenas $2,7{ }^{\circ} \mathrm{C}$.

Verifica-se que as temperaturas mais baixas coincidem com a época chuvosa, na qual predominam também os ventos mais fortes de origem Sul-Sudeste. Na época seca, as temperaturas são as mais elevadas; ao mesmo tempo, os ventos são um pouco mais fracos, com uma gama de distribuição mais variável em torno da direção Leste-Oeste.

A variação de salinidade apresenta uma relação bem estreita com a pluviometria, sendo os valores maiores correspondentes à época chuvosa. A média máxima foi de 360/00 e a mínima de 320/00 (Cavalcanti \& Kempf, 1970).

\section{MATERIAL E MÉTODOS}

\section{Em Campo}

Mensalmente, foi obtida uma amostragem representativa de indivíduos, provenientes de pescarias artesanais, utilizando-se covos e linha de fundo, nas praias do Pina, Boa Viagem, Piedade e Candeias, durante todo o ano por botes de pesca.

Os espécimens foram capturados durante o período de 17 meses, ou seja, de setembro/80 a janeiro/82. 


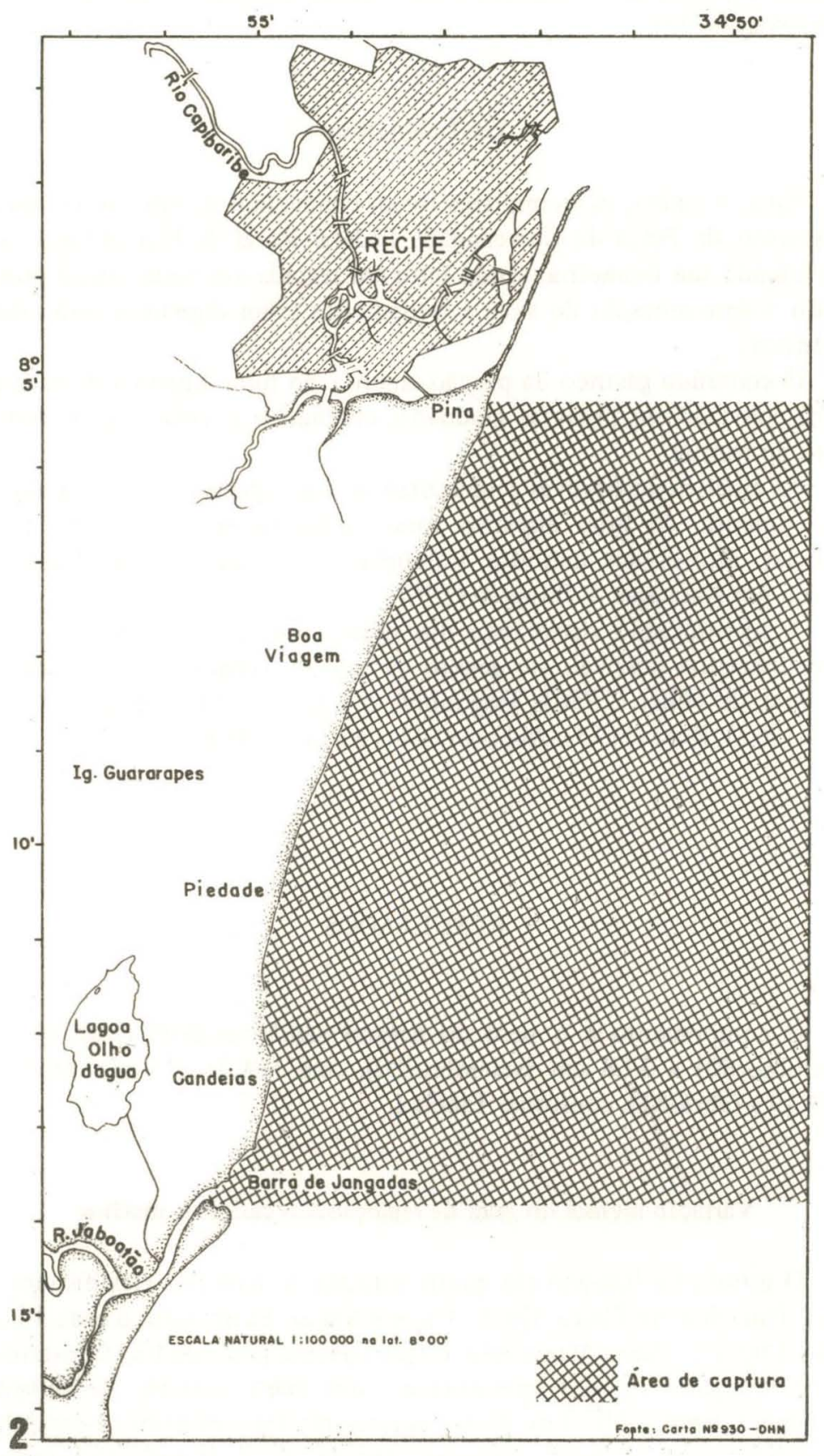

Fig. 2: Mapa da região onde foram realizadas as coletas. 
Analisou-se, individualmente, o conteúdo gástrico da porção anterior do tubo digestivo de 60 machos e 100 fêmeas, perfazendo um total de 160 peixes examinados.

\section{Em Laboratório}

Após a coleta, os exemplares foram conduzidos ao laboratório do Departamento de Pesca da Universidade Federal Rural de Pemambuco, onde era efetuada sua biometria, sendo feito em seguida um corte lateral possibilitando a determinação do sexo e retirada dos tubos digestivos para exames posteriores.

$\mathrm{O}$ conteúdo gástrico da porção anterior do tubo digestivo de cada peixe foi colocado em provetas graduadas obtendo-se o volume pelo deslocamento do formol.

As análises qualitativas e quantitativas dos organismos encontrados em cada conteúdo do tubo digestivo foram realizadas em microscópio e lupa binocular, através dos métodos Volumétrico e Freqüência de Ocorrência, descrito por Natarajan \& Jhingran (1981).

A identificação sistemática dos organismos encontrados foi feita na menor categoria possível, até gênero ou espécie, estando a mesma baseada nas obras de Miner (1950), Tregouboff \& Rose (1957), Yamaji (1959), Tinoco (1967), Rosa (1973), Abbott (1974) e'Rios (1975).

\section{RESULTADOS}

O comprimento e o peso do Balistes vetula variaram entre $19,3 \mathrm{~cm}$ (novembro/80) a $37,8 \mathrm{~cm}$ (setembro/80), com 184,31 g (novembro/80) a $1.199,59 \mathrm{~g}$ (abril/81), conforme Tabela I.

\section{Variação mensal do grau de repleção dos çangulos machos}

O estudo foi baseado em quatro estádios de repleção, classificados em: Cheio, Parcialmente Cheio, Quase Vazio e Vazio. Entretanto, o grau Parcialmente Cheio e Quase Vazio não foram comuns nos estudos. Constatou-se que os cangulos machos apresentaram uma maior ingestão de alimentos nos meses setembro/80, abril, junho, agosto, outubro, novembro e dezembro/ 81, quando todos os tubos digestivos estavam cheios de alimentos, apresentando um grau de repleção Cheio.

Com relação aos tubos digestivos vazios constatou-se uma incidência 


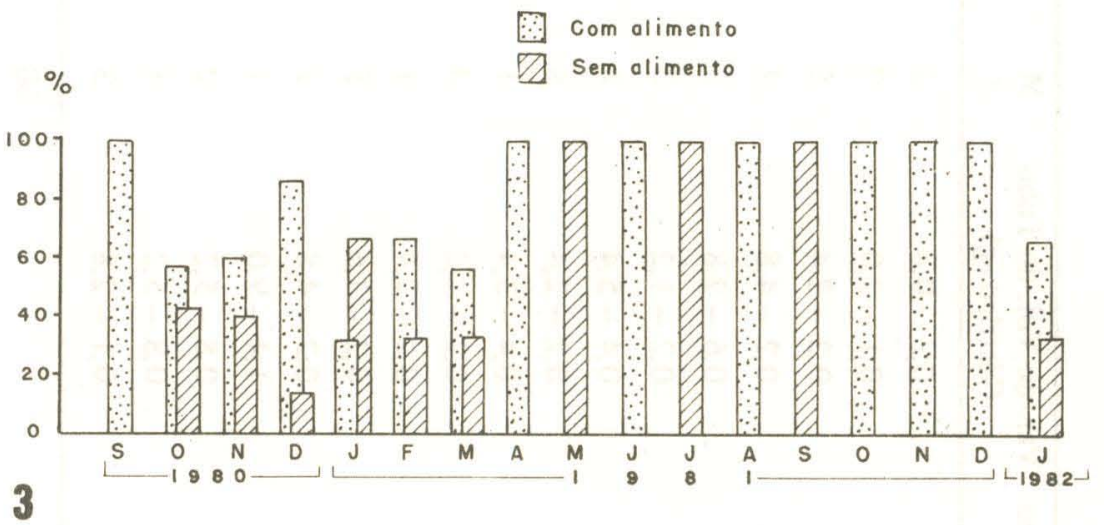

Fig. 3: Distribuição dos tubos digestivos com e sem alimento do Cangulo (ठ̋), Balistes vetula Linnaeus, 1758.

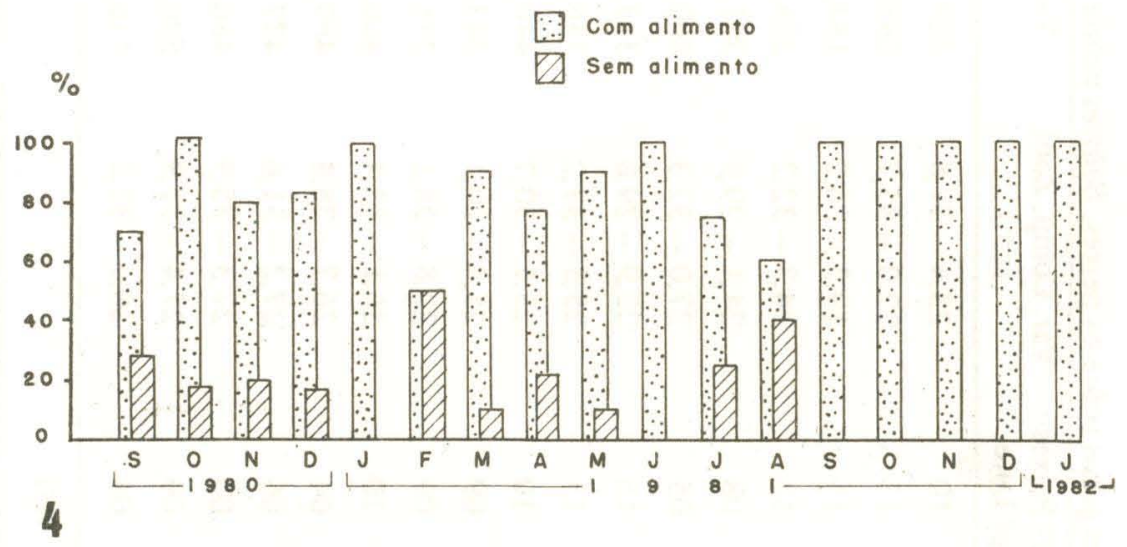

Fig. 4: Distribuição dos tubos digestivos com e sem alimento do Cangulo (\$), Balistes vetula Linnaeus, 1758. 
Tabela I - Dados gerais sobre os peixes, Balistes vetula.

\begin{tabular}{|c|c|c|c|c|c|c|c|}
\hline Meses & $\begin{array}{l}\text { No Peixes } \\
\text { p/ Lote }\end{array}$ & $\begin{array}{l}\text { Var. Comp. Zool. } \\
\text { (cm) }\end{array}$ & $\begin{array}{c}\text { Var. Peso Total } \\
\text { (g) }\end{array}$ & $\begin{array}{l}\text { Var. Peso do Tubo } \\
\text { Gástrico (g) }\end{array}$ & $\begin{array}{c}\text { Var. do Vol. do Tubo } \\
\text { Gástrico (g) }\end{array}$ & $\begin{array}{c}\mathrm{M} \\
0\end{array}$ & $\begin{array}{l}\mathrm{F} \\
\wp\end{array}$ \\
\hline Set. $/ 80$ & 10 & $23,4-37,8$ & $329,02-1173,07$ & $2,64-17,33$ & $0,2-0,8$ & 3 & 7 \\
\hline Out./80 & 13 & $22,0-35,7$ & $283,02-1036,71$ & $1,39-7,43$ & $0,1-2,0$ & 7 & 6 \\
\hline Nov./80 & 15 & $19,3-36,2$ & $184,31-1038,03$ & $2,12-8,72$ & $0,2-2,4$ & 5 & 10 \\
\hline Dez./80 & 13 & $24,3-32,2$ & $378,13-774,74$ & $1,83-12,88$ & $0,2-4,8$ & 7 & 6 \\
\hline Jan./81 & 08 & $24,1-29,9$ & $301,04-564,37$ & $3,57-9,92$ & $0,6-2,8$ & 3 & 5 \\
\hline Fev./81 & 05 & $27,0-32,3$ & $460,00-796,20$ & $3,61-10,66$ & $0,2-1,2$ & 3 & 2 \\
\hline Mar./81 & 16 & $22,9-29,8$ & $313,25-649,72$ & $2,89-11,65$ & $0,4-5,4$ & 7 & 9 \\
\hline Abr./81 & 15 & $25,3-37,2$ & $356,30-1199,59$ & $2,39-15,57$ & $0,2-2,4$ & 6 & 9 \\
\hline Mai./81 & 10 & $25,5-30,7$ & $440,01-581,58$ & $4,97-16,27$ & $0,4-3,4$ & 1 & 9 \\
\hline Jun./81 & 09 & $25,4-29,1$ & $343,20-578,06$ & $3,81-11,19$ & $0,2-2,2$ & 4 & 5 \\
\hline Jul./81 & 09 & $22,8-28,1$ & $275,98-576,73$ & $0,27-7,29$ & $0,2-2,4$ & 1 & 8 \\
\hline Ago./81 & 10 & $26,1-33,4$ & $406,04-842,99$ & $3,17-10,98$ & $0,2-3,6$ & 5 & 5 \\
\hline Set./81 & 04 & $26,7-28,4$ & $469,82-588,78$ & $5,07-14,49$ & $0,2-4,8$ & 1 & 3 \\
\hline Out./81 & 05 & $27,2-31,6$ & $477,36-919,06$ & $15,14-25,49$ & $1,4-9,0$ & 1 & 4 \\
\hline Nov./81 & 05 & $27,3-35,9$ & $480,92-987,58$ & $4,94-18,58$ & $0,8-5,2$ & 2 & 3 \\
\hline Dez./81 & 09 & $27,4-31,9$ & $500,55-818,45$ & $10,10-27,35$ & $0,8-5,2$ & 1 & 8 \\
\hline Jan./82 & 04 & $26,1-32,2$ & $416,44-707,64$ & $2,12-15,16$ & $0,1-2,8$ & 3 & 1 \\
\hline Total & 160 & & & & & 60 & 100 \\
\hline
\end{tabular}


total dos exemplares coletados nos meses: maio, julho e setembro/81 (Fig. $3)$.

\section{Variação mensal do grau de repleção dos cangulos fêmeas}

No que diz respeito aos cangulos fêmeas, observou-se uma dominância durante todo o estudo dos tubos digestivos Cheios de alimentos, principalmente nos meses de janeiro, junho, setembro, outubro, novembro, dezembro/81 e janeiro/82.

Ocorreram também em vários meses exemplares sem nenhuma ingestã de alimentos, tendo, portanto, o seu grau de repleção Vazio, como se podt observar na Figura 4.

\section{Principais organismos encontrados no tubo digestivo dos machos}

O exame do tubo digestivo dos exemplares machos de Balistes vetula revelou a presença dominante dos seguintes organismos: Moluscos, destacaram-se pela sua abundância e freqüência chegando a alcançar 50,0\% (janeiro/ 81), sobre os demais tipos de alimento (Fig. 5), sobressaindo-se pelo número de gêneros. Entre os Scaphopoda foram registrados a presença de Dentalium e Cadulus; entre os Bivalvos foram comuns os gêneros Chama e Anadara e os Gastrópodos dos gêneros Calliostoma, Nassarius, além de outros não identificados (Tabela II); Crustáceos também estiveram presentes em quase todo período de estudo, destacando-se principalmente por isópodos da família Aegidae, espécie Rocinela signata, além de um alto percentual de restos digeridos de crustáceos (44,4\%); conforme Tabela II; Peixes da Classe Osteichthyes também foram frequentes, porém menos abundantes com relação aos organismos anteriores; Foraminíferos tiveram uma marcante participação, destacando-se a familia Soritidae, com as espécies Archaias angulatus e Peneroplis proteus; Equinodermos também estiveram presentes, sobressaindo-se os restos semi-digeridos, notadamente de ouriços-domar; outros organismos estiveram representados principalmente por Briozoários, Esponjas, Poliquetos, Nematóides, Celenterados, Braquiópodos e Algas (Fig. 5 e Tab. II).

A presença abundante de restos digeridos de animais e a ausência das partes duras dificultaram o reconhecimento dos grupos no conteúdo alimentar.

Vale salientar a presença de sedimentos (grãos-de-areia) na maioria dos tubos digestivos analisados, comprovando um hábito alimentar essencialmente bêntico para esses peixes.

Principais organismos encontrados no tubo digestivo dos canguios têmeas

O exame do tubo digestivo dos exemplares fêmeas de Balistes vetula revelou a presença dominante dos seguintes organismos: Moluscos, presentes 


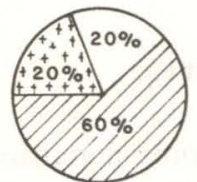

SET. $/ 80$

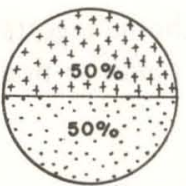

J AN./ 8 |

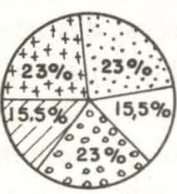

JUN./8I

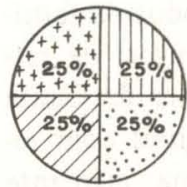

DEZ./81

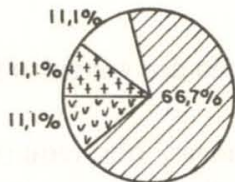

OUT. $/ 80$

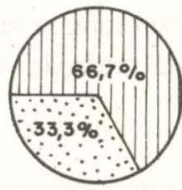

FEV./81

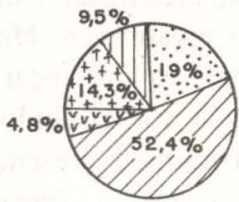

AGO./81

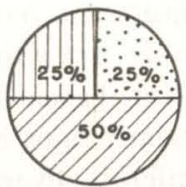

JAN. $/ 82$

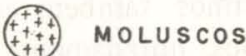

(II) PEIXES

$\because \quad$ CRUSTÁCEOS

(2) EQUINODERMOS

$\therefore$ FORAMINIIFEROS

OUTROS ORGANISMOS

SEDIMENTOS

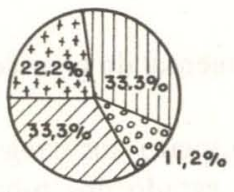

NOV./8O

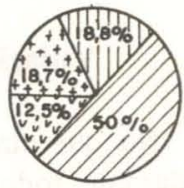

DEZ.180

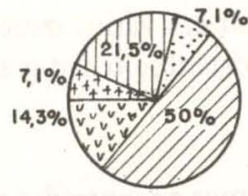

MAR./8I

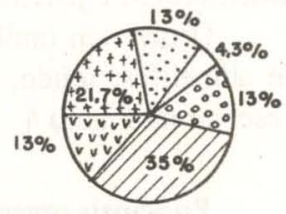

ABR./81

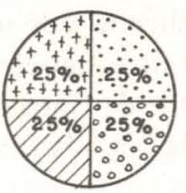

OUT./8I

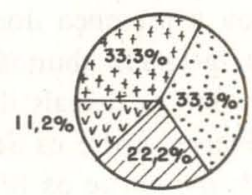

NoV./81

Fig. 5: Itens alimentares encontrados no tubo digestivo do Cangulo (ठే), Balistes vetula Linnaeus, 1758. 
Tabela II - Freqüência de Ocorrência dos itens alimentares do cangulo (ठ), Balistes vetula Linnaeus, 1758.

\section{MOLUSCOS:}

Escafópodos do gênero Dentalium $\quad 24,4$

Escafópodos do gênero Cadulus $\quad 24,4$

Bivalvos do gênero Chama 13,3

Gastrópodos do gênero Calliostoma 2,2

Gastrópodos do gênero Nassarius $\quad 4,4$

Gastrópodos não identificados 20,0

Restos de Bivalvos (Anadara spp.) 11,1

Conchas de Bivalvos 13,3

Restos 28,9

CRUSTÁCEOS:

Isópodo da familia Aegidae, Rocinela signata $\quad 4,4$

Copépodos 2,2

Restos 44,4

PEIXES:

Restos de peixe da Classe Osteichthyes $\quad 40,0$

Ovos $\quad 4,4$

Escamas $\quad 4,4$

PROTOTOA:

Foraminifero da famnlia Soritidae, Archaias angulatus $\quad 15,6$

Foraminífero da familia Soritidae, Peneroplis proteus $\quad 4,4$

EQUINODERMOS:

Espinhos de ouriço $\quad 6,7$

Restos 11,1

BRIOZOARIOS:

$\begin{array}{ll}\text { Não identificados } & 17,8\end{array}$

ESPONJAS:

Restos 11,1

ANELIDEOS:

Poliquetos $\quad 4,4$

Tubos $\quad 4,4$

VERMES:

Nematóides $\quad 4,4$ 
Tabela II - Continuação.

Alimentos

CELENTERADOS:

Hidróides 2,2

Corais 2,2

BRAQUIÓPODOS:

Não identificados $\quad 2,2$

ALGAS:

Algas do gênero Halimeda $\quad 2,2$

$\begin{array}{ll}\text { Algas calcáreas ñ̃o identificadas } & 26,7\end{array}$

OUTROS ORGANISMOS:

Restos de insetos

Ovos planctônicos $\quad 6,7$

\begin{tabular}{ll} 
Restos de animais $\quad 46,7$ \\
\hline
\end{tabular}

SEDIMENTOS (grãos-de-areia)

em quase todo período de estudo, com ocorrência máxima de $27,3 \%$ no mês de agosto/81. Entre os Scaphopoda destacaram-se os gêneros Dentalium e Cadulus; já os Bivalvos estiveram representados pelos gêneros Chama, Pecten, Argopecten, Anadara, Hiponix e pela espécie Tellina sybaritica, além de fragmentos de Pteropoda, ocorrendo uma grande incidência de gastrópodos não identificados (Tab. III); Crustáceos, representados por isópodos da família Aegidae, espécie Rocinela signata, restos de anfípodos, além de restos de crustáceos; Peixes tiveram uma ocorrência secundária chegando a alcançar $71,4 \%$ (dezembro/80), sobre os demais grupos alimentares (Fig. 6); Equinodermas presentes em quase todo o estudo, destacando-se restos de estrelado-mar, holotúria e ouriços-do-mar; Foraminíferos foram comuns os da familia Soritidae e as seguintes espécies, Archaias angulatus, Peneroplis proteus (Tab. III).

Os outros organismos participaram como alimentos secundários, na alimentação desses peixes, isto é, Briozoários, Esponjas, Anelídeos, Vermes, Celenterados, Algas e restos de animais. Entretanto, este item alimentar alcançou altos índices durante os meses de estudo, compreendendo uma variação de 18,2\% (agosto/81) a 100\% (fevereiro/81 e janeiro/82) (Fig. 6).

Também foi registrada a presença de sedimentos (grãos-de-areia), no tubo digestivo desses peixes.

\section{CONSIDERAÇÕES GERAIS E CONCLUSÕES}

A análise quanto-qualitativa do conteúdo dos tubos digestivos dos 
Tabela III - Freqüência de Ocorrência dos itens alimentares do cangulo (\$), Balistes vetula Linnaeus, 1758.

MOLUSCOS:

Escafópodos do gênero Dentalium $\quad 15,5$

Escafópodos do gênero Cadulus $\quad 15,5$

Bivalvos do gênero Chama $\quad 3,6$

Bivalvos do gênero Pecten $\quad 1,2$

Bivalvos do gênero Argopecten $\quad 1,2$

Bivalvos do gênero Anadara $\quad 10,7$

Bivalvos do gênero Hiponix $\quad 2,4$

Bivalvos da espécie Tellina sybaritica $\quad 1,2$

Vários fragmentos do gênero Pteropoda $\quad 4,8$

Conchas de Gastrópodos $\quad 2,4$

Gastrópodos não identificados $\quad 21,4$

Conchas de bivalvos $\quad 17,9$

Opistobranchia não identificados $\quad 1,2$

Restos 38,1

CRUSTÁCEOS:

Isópodo da família Aegidae, Rocinela signata $\quad 3,6$

Larvas $\quad 1,2$

Restos de anfípodos 2,4

Olhos $\quad 12,0$

Restos 38,1

PEIXES:

Restos de peixe da Classe Osteichthyes $\quad 39,3$

Escamas $\quad 6,0$

PROTOZOA:

Foraminíferos da família Soritidae, Archaias angulatus 26,2

Foraminíferos da família Sorotidae, Peneroplis proteus $\quad 9,5$

EQUINODERMOS:

Estrelas-do-mar (restos) $\quad 2,4$

Holotúria (restos) $\quad 1,2$

Espinhos-de-ouriços $\quad 9,5$

Restos $\quad 14,3$

BRIOZOÁRIOS:

Não identificados $\quad 16,7$

ESPONJAS:

Não identificados $\quad 13,1$

ANELÍDEOS:

$\begin{array}{ll}\text { Poliquetos não identificados } & 10,7\end{array}$ 
Tabela III - Continuação.

Alimentos

$\%$

VERMES:

Nematóides

CELENTERADOS:

Hidróide

Corais

Restos

BRAQUIÓPODOS:

Não identificados

ALGAS:

Algas do gênero Halimeda

Algas calcáreas não identificadas

Talos de macroalgas

OUTROS ORGANISMOS:

Restos de insetos

Ovos

Restos digeridos de animais

peixes (Balistes vetula) revelou uma ocorrência de diversos organismos, os quais atingiram altos índices de animais, notadamente os moluscos, crustáceos e peixes.

Os itens alimentares mostraram uma dieta carnívora de natureza bentônica, a exemplo do que ocorre com outros membros desta mesma espécie (Randall, 1968 e Menezes, 1981).

Dos exemplares examinados $61,88 \%$ eram fêmeas e $38,12 \%$, machos.

Entre os grupos observados, os alimentos considerados essenciais são os moluscos das Classes Scaphopoda, Bivalvia e Gastropoda tanto para os machos como para as fêmeas; entretanto, outros grupos podem ser mencionados como os restos semi-digeridos de crustáceos e peixes. Ocorreram em quantidades significativas em ambos os sexos, vários gêneros de moluscos, sobressaíndo-se: Dentalium, Cadulus (Scaphopoda); Chama, Anadara (Bivalvia). Entretanto, além desses gêneros, as fêmeas ingeriram moluscos dos gêneros: Pecten, Argopecten, Hiponix, Tellina

Outros grupos de animais ocorreram, também, secundariamente: Equinodermos, Foraminíferos, Briozoários, Esponjas, Anelídeos, Vermes, Celenterados, Braquiópodos, fazendo parte da alimentação do cangulo.

Apesar do Balistes vetula ser um peixe eminentemente camívoro, foi verificado um relativo percentual de algas calcáreas, principalmente as cloro- 


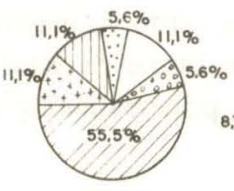

SET./BO

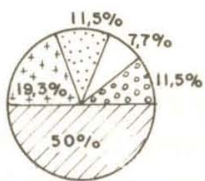

JAN./BI

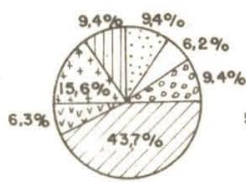

MAIO/8I

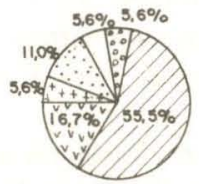

SET./8I

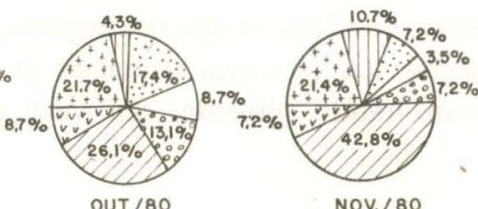

NoV. $/ 80$

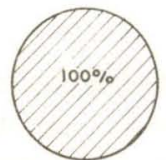

FEV./8I

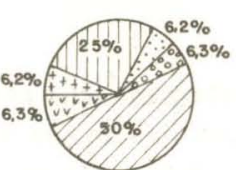

MAR. /81

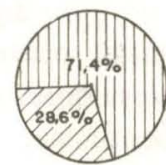

DEZ. $/ 80$

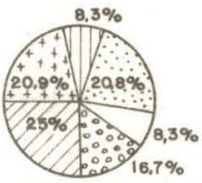

ABR./81
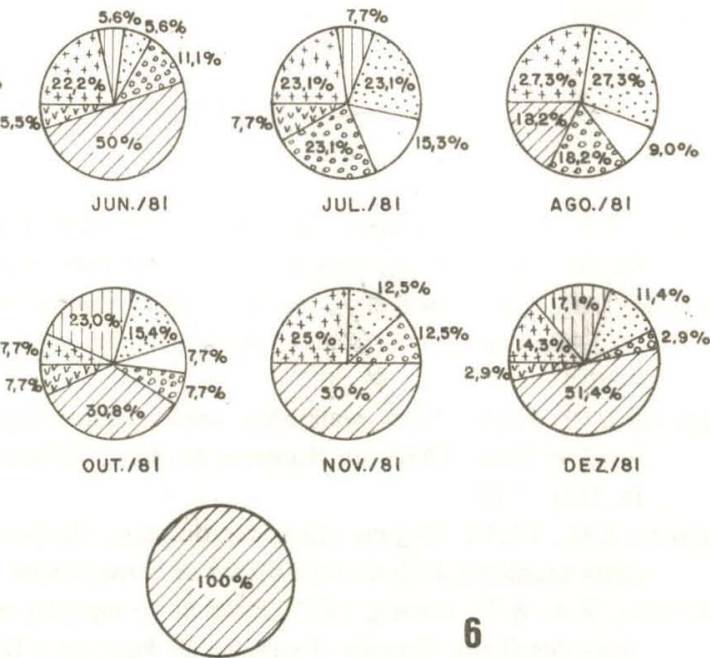

JAN. / 82

6

Fig. 6: Itens alimentares encontrados no tubo digestivo do Cangulo ( $($ ), Balistes vetula Linnaeus, 1758. 
fíceas do gênero Halimeda no bolo alimentar.

Não foi observada no período estudado preferência por determinados tipos de alimentos em decorrência de sexos, salvo as fêmeas ingerirem maiores variedades de moluscos, não se constatando também diversificação alimentar, levando em consideração as épocas do ano e comprimento dos exemplares.

Devido a grande incidência de animais encontrados no conteúdo gástrico do tubo digestivo, verificou-se que os cangulos, tanto os machos como as fêmeas, provenientes das pescarias artesanais do litoral de Recife - PE, são possuidores de um hábito alimentar carnívoro, principalmente de natureza bentônica.

\section{AGRADECIMENTOS}

Os autores externam seus agradecimentos aos Professores Ivan Medeiros Tinoco, Deusinete de Oliveira Tenório, José Audísio Costa Luna, Roberto de Albuquerque Régis, Dinalva de Souza Guedes, Libânia Maria Maia Rodrigues Couto e Cileide Maria Acioli Soares, pela ajuda na identificação do material.

\section{REFERENNCIAS}

Abbott, R.T., 1974. American seashells. In: The marine Mollusca of the Atlantic and Pacific coast of North America. 2. ed. New York, Van Nostrand, 661 p.

Barroso, L.M., 1967. Biologia e pesca do peixe voador (Hirundicthys affinis Gunther) no Estado do Rio Grande do Norte. Boletim de Estudos de Pesca, Recife. 7(1): 9-37.

Barroso, L.M., 1965. . Nota preliminar sobre a alimentação do Xaréu preto (Caranx lugubris Poey, 1960) no Nordeste do Brasil. Boletim de Estudos de Pesca, Recife, 5(5): $7-11$.

Barroso, L.M., 1965a. Regime alimentar do pargo (Lutjanus aya Bloch, 1795) no Nordeste brasileiro. Boletim de Estudos de Pesca, Recife, 5(3): 7-11.

Carvalho, V.A. \& R. Branca, 1977. Relação de espécies marinhas e estuarinas do Nordeste brasileiro. Brasılia, Programa de Pesquisa e Desenvolvimento Pesqueiro do Brasil, 60 p.

Cavalcanti, L.B. \& M. Kempf, 1970. Estudo da plataforma continental na área do Recife (Brasil). 2 Meteorologia e Hidrologia. Trabalhos oceanográficos da Universidade Federal de Pernambuco, Recife, 9/11: 149-157.

Cervigon, F, 1966. Los peces marinos de Venezuela. Caracas, Estación de Investigaciones Marinas de Margarita, v.2.

Eskinazi-Leça, E.; A.L. Vasconcelos-Filho \& J.E. Silva, 1976. Aspectos gerais sobre a alimentação de peixes Mugillídeos, ocorrentes no Canal de Santa Cruz, Pernambuco, Brasil. Anais Instituto Biologia da UFRPE, Recife, 1: 143-155.

Furtado, E. \& M.F. Menezes, 1972. Alimentação do pargo Lutjanus purpureus Poey, no Nordeste brasileiro. Arquivos de Ciências do Mar, Fortaleza, 12(2): 105-108. 
Greenwood, P.H.; D.E. Rosen; S.H. Weitzman \& G.S. Myers, 1966. Phyletic studies of teleostean fishes, with a provisional classification of living forms. Bulletin of the American Museum of natural History, New York, 131(4): 341-455.

Guedes, D. de S. \& A.L. Vasconcelos Filho, 1980. Estudo ecológico da Região de Itamaracá, Pernambuco, Brasil. IX Informações sobre a alimentação dos Bagres Branco e Amarelo (Pisces Ariidae). Trabalhos Oceanográficos da Universidade Federal de Pernambuco, Recife, 15: 323-330.

Herald, E.S., 1962. Los Peces. Barcelona, Ed. Seix Barral, 352 p.

Jordan, D.S. \& B.W. Evermann, 1896. The fishes of North and middle America: a descriptive catalogne of the afecus of fishlike vertebrates jound in the watus of North America, North of the Isthmus of Panamá. Bulletin of the United States National Museum, Washington, 47(1/4).

Lima, H.H. \& A.M.E. Oliveira, 1978. Segunda contribuição ao conhecimento dos nomes vulgares de peixes do Nordeste brassileiro. Boletim Ciências do Mar, Fortaleza, (29): 1-26.

Menezes, M.F., 1969. Alimentação da cavala, Scomberomonus cavala (Cuvier), em águas costeiras do Estado do Ceará. Arquivos de Ciências do Mar, Fortaleza, 9(1): 15-20.

Menezes, M.F., 1970. Alimentação da serra, Scomberomorus maculatus (Mitchill), em águas costeiras do Estado do Ceará. Arquivos de Ciências do Mar, 10(2): 171-176.

Menezes, M.F., 1981. Aspectos da biologia e biometria do Cangulo, Balistes vetula Linnaẻus, no Nordeste do Brasil. In: CONGRESSO BRASILEIRO DE ENGENHARIA DE PESCA, 2, Recife, Anais. Recife, Associação dos Engenheiros de Pesca de Pernambuco, p. 438-501.

Menezes, M.F., 1968. Sobre a alimentação do camurupim Tarpon atlanticus (Valenciennes) no Estado do Ceará. Arquivos da Estação Biologia Marinha da Universidade Federal do Ceará, 8(2): 145-149.

Menezes, M.F. \& L.P. Aragão, 1977. Biometria e biologia do bonito, Euthunnus alletteratus (Rafinesque), no Estado do Ceará, Brasil. Arquivos de Ciências do Mar, Fortaleza, 17(2): 95-100.

Miner, R.W., 1950. Field book of seashore life. New York, Putmann, 888 p.

Moura, R.T. de., 1981. Sobre a fecundidade, desova e tamanho da primeina maturação sexual do cangulo Balistes vetula (Linnaeus, 1758), Recife. 23 p. MonografiaEngenharia de Pesca-UFRPE.

Natarajan, A.V. \& A.G. Jhingran, 1961. Index of preponderance - a method of grading the food elements in the stomach analysis of fishes. Indian Journal of Fisheries, New Delhi, 8(1): 54-9.

Randall, J.E., 1968. Caribbean reef fishes. Hawaii, Universit of Hawaii. p. 258-9.

Rosa, C.N., 1973. Os animais de nossas praias. 2. ed. São Paulo, EDART, 187 p.

Ribeiro, A.M., 1915. Fauna Brasiliense. Peixes (Eleutherobranchios Aspirophoros). Arquivos Museu Nacional. Rio de Janeiro, 17. 1-827.

Rios, E.C. 1975. Brazilian marine mollusks iconography. Rio Grande, Museu Oceanográfico, $331 \mathrm{p}$.

Santos, E., 1952. Nossos peixes marinhos: vida e costumes dos peixes do Brasil. Rio de Janeiro, F. Briguiet, p. 215-6.

Silva, J.E. \& A.L. Vasconcelos Filho., 1972. Aspectos Gerais sobre a alimentação de camorins (Centropomus undecimalis, Bloch e Centropomus parallelus, Poey). Anais do Instituto de Biologia, UFRPE, Recife, 2: 33-41. 
Smith, J.L.B., 1961. The sea fishes of southern A frica. Cape Town, Central News Agency. $580 \mathrm{p}$.

Soares-Filho, P.L. da C., 1980. Relação peso/comprimento e alguns aspectos da reprodução de Balistes vetula (Linnaeus, 1758) Pisces-Tetraodontiformes Balistidae. Recife, 49 p. Monografia-Engenharia de Pesca - UFRPE.

Soares, W.J. de M., 1981. Estudo preliminar da população do cangulo Balistes vetula (Linnaeus, 1758) (Tetraodontiformes-Balistidae) e alguns informes sobre a reprodução. Recife, 68 p. Monografia-Engenharia de Pesca - UFRPE.

Tinoco, I.M., 1967. Foraminíferos do Atol das Rocas. Trabalhos Oceanográficos da Universidade Federal de Pernambuco, Recife, 7/8: 91-114.

Tregouboff, G. \& M. Rose., 1957. Manual de Planctonologie Mediterraneenne. Paris, Centre National de La Recherche Scientifique, v. 1.

Vasconcelos Filho, A. de L., 1979. Estudo Ecológico da Região de Itamaracá, Pemambuco, Brasil. IV Alimentação da sardinha-bandeira, Opisthonema oglinum Le Sueur, 1817 no Canal de Santa Cruz, Trabalhos Oceanográficos da Universidade Federal de Pernambuco, Recife, 14: 105-106.

Vasconcelos Filho, A. de L. \& E.M.B. Galiza, 1980. Hábitos alimentares dos peixes Centropomídeos cultivados em viveiros da regiāo de Itamaracá, Pernambuco. Revista Nordestina de Biologia, João Pessoa, 3 (especial): 111-122.

Vasconcelos Filho, A. de L.; M.L.C. Alves \& E. Eskinazi-Leça, 1981. Estu do Ecológico da Região de Itamaracá, Pernambuco, Brasil. XVIII Aspectos gerais sobre a alimentação da carapeba listrada, Eugerres spp. e da carapeba prateada, Diapterus spp. (Pisces-Gerreidae), no Canal de Santa Cruz, Recife. Trabalhos Oceanográficos da Universidade Federal de Permambuco, 16: 89-104.

Yamaji, J., 1959. The plancton of Japaneses Coastal Waters. Osaka, Hoikusha. 230 p. 